probable value of T-score in patients with known rates of calcification of the coronary and carotid arteries were obtained by regression analysis. These factors were equivalent density of coronary calcinoses $(p=0,0046)$, the volume of the carotid calcifications $(p=0,0039)$, the mass of calcifications of the carotid arteries $(p=0,0054)$ and the presence of a stenosis of the carotid arteries $(p=0.0001)$. The predictive model for estimating the probable value of T-score has been is designed using regression coefficients of each of the factors. The value is equal to the Fisher statistic $F=9,52$, $p$-value $<0.000001$, multiple correlation model's coefficient is 0.753 .

Conclusions: The results of this study indicate that rates of calcification of the carotid and coronary arteries, resulting in the planned survey on MSCT patients with multifocal atherosclerosis have a high predictive capacity for assessing the probable value of the T-score and early detection of osteopenic syndrome in these patients.

Disclosure of Interest: None declared

DOI: 10.1136/annrheumdis-2017-eular.5654

\section{FRI0537 PREVENTION STRATEGY OF OSTEOPOROTIC FRACTURES IN PORTUGAL: AN ANALYSES ON A COHORT OF HIP FRACTURED PATIENTS}

A. Daniel ${ }^{1}$, M.L. Marques ${ }^{1}$, L. Brites ${ }^{1}$, C. Torres $^{2}$, A. Marques ${ }^{1}$, J.A. Pereira da Silva ${ }^{1} \cdot{ }^{1}$ Rheumatology Unit, Centro Hospitalar e Universitário de Coimbra; ${ }^{2}$ Rheumatology Unit, Faculdade de Medicina da Universidade de Coimbra, Coimbra, Portugal

Background: Despite the fact that Portugal has one of the lowest rates of hip fractures in Western Europe, more than 10.000 patients are admitted every year to the Portuguese National Health Service due to hip fragility fractures. The burden of the problem will tend to increase in coming years, unless effective preventive measures are put in place. ${ }^{1}$

Objectives: The aim of our work was to evaluate the percentage of patients under osteoporotic treatment (OT) before and after a hip fracture (HF) and compare these results with the percentage of patients who should be under treatment, according to FRAX model and Portuguese cost-effectiveness guidelines (PG) for OT. ${ }^{1}$

Methods: Patients diagnosed with a HF, between May 1st and October 31st 2013, from a single tertiary hospital, were included in this transversal study. Patients or their primary caregiver were contacted by phone to gather data regarding demographic and clinical features, including risk factors for Osteoporosis (OP) defined by FRAX ${ }^{\circledR}$. Clinical data was obtained from medical files.

FRAX ${ }^{\circledR}$ without mineral bone density was used to calculate the 10 year fracture risk. For each patient the $F R A X^{\circledR}$ was scored according to data available the day before the present $\mathrm{HF}$ (ie the current fracture was not considered as a previous fracture for the purpose of risk prediction). Thresholds for therapeutic intervention were defined according to PG: a 10-year probability of a major osteoporotic fracture (OF) $\geq 11 \%$ and/or a 10 year probability of $\mathrm{HF} \geq 3 \%{ }^{1}$.

Results: The mean age of the population $(n=130)$ was $81.6 \pm 8.6$ years, and $69.2 \%$ were female. Before the current HF, only $23(17.7 \%)$ of the patients had been prescribed some form of medication for OP: bisphosphonates $(n=2)$, strontium ranelate $(n=3)$ and calcium + vitamin D supplementation $(n=13)$; the other patients or caregivers didn't specify the ongoing medication.

About 65 patients had a previous fracture, of which 8 patients had $\geq 1$ fragility fracture of the hip; 6 had $\geq 1$ symptomatic vertebral fragility fracture and 16 had $\geq 2$ fragility fractures, independently of the site of the fracture. According to $P G$, all these $30(26 \%)$ patients should be under OT without the need for FRAX ${ }^{\circledR}$ risk calculation.

The mean 10 -year major OF probability was $21.2 \pm 14 \%$ and the mean 10 -year HF probability was $13.7 \pm 12.9 \%$. According to FRAX ${ }^{\circledR}, 104(80 \%)$ of the patients had indication to start OT based on the 10-year risk of major OF and $117(90 \%)$ based on the 10-year risk of HF.

After hospitalization, although all the patients had formal indication for treatment, only $11(8.5 \%)$ patients had received a prescription for OT up to one year after the fracture event.

Conclusions: Similar to other countries, the percentage of patients under OT (before and after HF) in Portugal is extremely low. Risk estimation by FRAX ${ }^{\circledR}$ and application of current PG would allow clinicians to identify these patients and introduce appropriate preventive measures. Continued efforts are needed to promote timely prevention, most especially after the first fragility fracture.

\section{References:}

[1] Marques A, Rodrigues AM, Romeu JC et al. Multidisciplinary Portuguese recommendations on DXA request and indication to treat in the prevention of fragility fractures. Acta Reumatol Port 2016;41:305-321.

Disclosure of Interest: None declared

DOI: 10.1136/annrheumdis-2017-eular.3277

\section{FRI0538 EVALUATION OF FACTORS THAT INCREASE FRACTURE RISK IN BREAST CANCER}

A. Anand ${ }^{1}$, M. Ghafouri ${ }^{2}$, M. Bukhari ${ }^{1} .{ }^{1}$ University Hospitals of Morcambe Bay NHS Foundation Trust; ${ }^{2}$ Lancaster University, Lancaster, United Kingdom

Background: Women with breast cancer are at an increased risk of fractures.
This is present in patients with both active and treated disease. In addition to established risk factors of fractures, patients with breast cancer are exposed to additional factors that further compromise bone strength. These factors primarily include: the malignancy interfering with bone metabolism and breast cancer treatments inducing bone loss.

Objectives: To evaluate fracture risk in active and treated breast cancer patients, and to understand the role bone mineral density (BMD) plays in predicting fracture risk.

Methods: The study population included breast cancer patients with active and treated disease referred to dual-energy X-ray absorptiometry (DEXA) scanning at the Royal Lancaster Infirmary between 2004-2015. Patients on aromatase inhibitors were excluded because of its' negative effect on oestrogen.

From this population, we collected BMD measurements of the femur and lumbar vertebra. Alongside information on physical characteristics such as age, height, weight, body mass index (BMI), average tissue thickness, lean and fat mass were measured.

To evaluate other precipitating factors known to increase fracture risk we included: smoking status, steroids use, alcohol, family history of fractures, diagnosis of rheumatoid arthritis and secondary osteoperosis.

Data analysis was done on R 3.3.2 software. Odds ratios were calculated using logistic regression and age adjusted models were compared using the likelihood ratio test. Categorical data was analysed using Chi squared and Fischer's exact test, while continuous data was analyzed using t-test.

Results: The study population was a total of 306 patients with a mean age of 63.6 years. 146 of the study group had active disease, while 160 patient were breast cancer survivors. Of the total population $87(28 \%)$ had sustained at least one fracture.

Active breast cancer insignificantly increased fracture risk in comparison to the cancer survivor population $(O R=1.330,95 \% C l=0.801-2.218, p=0.271$ ).

Physical characteristics that significantly increased fracture risk included increased age and decreased average fat percentage (Table 1). BMD reduction in the femoral neck and all vertebrae significantly increased odds of having a fracture (Table 2). External factors such as smoking status, alcohol consumption, family history and steroid use had no significant effect.

Table 1. Physical Characteristics and Fracture Risk

\begin{tabular}{lccc}
\hline & Odds Ratio & 95\% Confidence Interval & $p$-value \\
\hline Age & 1.407 & $1.106-1.803$ & 0.043 \\
BMI & 0.832 & $0.639-1.074$ & 0.159 \\
Average Tissue Thickness & 0.929 & $0.847-1.016$ & 0.108 \\
Average Fat Percentage & 0.069 & $0.005-0.883$ & 0.040 \\
Fat Mass & 0.997 & $0.994-0.999$ & 0.032 \\
Lean Mass & 1.003 & $1.000-1.006$ & 0.032 \\
\hline
\end{tabular}

Table 2. BMD Results from DEXA Scan

\begin{tabular}{lccc}
\hline & Odds Ratio & 95\% Confidence Interval & $p$-value \\
\hline Left Neck of Femur & 0.035 & $0.035-0.309$ & 0.002 \\
Right Neck of Femur & 0.063 & $0.006-0.561$ & 0.013 \\
Lumber Vertebra Total (L1-4) & 0.063 & $0.013-0.285$ & 0.0002 \\
\hline
\end{tabular}

Conclusions: In conclusion, this study emphasizes DEXA measurements are the best predictive tool for fractures in breast cancer patients. Thus further supporting the need for increased BMD surveillance for those diagnosed with breast cancer who are not on aromatase inhibitors.

References:

[1] Body J: Increased fracture rate in women with breast cancer: a review of the hidden risk. Body BMC Cancer 2011, 11:304.

[2] R Core Team (2016). R: A language and environment for statistical computing. R Foundation for Statistical Computing, Vienna, Austria.

Disclosure of Interest: None declared

DOI: 10.1136/annrheumdis-2017-eular.3829

\section{FRI0539 IMPACT OF THE IMPLEMENTATION OF A FRACTURE LIAISON SERVICE IN PHARMACEUTICAL EXPENSES FOR OSTEOPOROSIS}

A. Naranjo $^{1}$, S. Ojeda ${ }^{1}$, S. Rodriguez ${ }^{2}$, I. Bernardos ${ }^{1}$, A. Molina ${ }^{1}$, F. de la Nuez $^{3}$, M. Negrin ${ }^{4} .{ }^{1}$ Rheumatology; ${ }^{2}$ Geriatrics, Hospital Univ. Gran Canaria Dr. Negrin; ${ }^{3}$ Rational Drug Use Service, Servicio Canario de la Salud; ${ }^{4}$ Met. cuantitativos en Economia, Universidad de Las Palmas, Las Palmas de Gran Canaria, Spain

Background: In 2012 a Fracture Liaison Service (FLS) was implemented in Hospital Dr Negrin

Objectives: To analyze the economic impact of the FLS on pharmaceutical expenditure for osteoporosis.

Methods: Expenditure on osteoporosis medication (government inpunt) was collected from January 1th, 2011 to October 1th, 2016. The data distinguish group I (calcium and vitamin D), group II (Bisphosphonates, denosumab, SERM, strontium and teriparatide) and total expenditure (sum of groups I and II). Intravenous bisphosphonates were not included.

Gran Canaria island is organized in two health areas; the North and the South areas. The population aged $>65$ years in the North and South areas in 2016 were 